\title{
Developmental Dysplasia of the Hip: Optimal Ultrasound Screening Strategy Among High Risk Newborns
}

\author{
Mohammed Shawky Abdullah, Ashraf Anas Zytoon \\ Radiology Department, Faculty of Medicine, Menoufiya University Menoufiya, Egypt
}

Email address:

m.shawky76@yahoo.com (M. S. Abdullah)

\section{To cite this article:}

Mohammed Shawky Abdullah, Ashraf Anas Zytoon. Developmental Dysplasia of the Hip: Optimal Ultrasound Screening Strategy Among High Risk Newborns. International Journal of Medical Imaging. Vol. 3, No. 3, 2015, pp. 49-58. doi: 10.11648/j.ijmi.20150303.12

\begin{abstract}
Musculoskeletal ultrasonography is becoming an attractive imaging modality for screening of high risk infants for DDH because of its advantages such as accessibility, low cost, rapid examination and ability to detect the soft tissues \& cartilage which cannot be seen by conventional radiography. Hip ultrasonography has the ability not only to visualise the unossified parts of the newborn hip but also to show movement of the femoral head within the socket. Ultrsonographic screening of all newborns can identify cases at an earlier age and lead to earlier treatment and prevent complication. So we conclude that the hip ultrasound screening of high risk infants for developmental dysplasia of the hip is important for early diagnosis \& treatment. The current study included 270 cases of high risk infants for DDH. Their ages ranged from 4 weeks to 6 months. We found that the most prevalent risk factor for hip dislocation in our study was ceaserean section delivery followed by breech presentation and oligohydraminos. The mean value of Alpha angle of the right hip was high than that of the left hip, where mean values of Beta angle of left hip was high than that of right hip. Graf type degree Ia was the most frequent degree in the right \& left hips followed by IIa in right hip and IIb in left hip. Caesarean section delivery was the most prevalent risk factor in Graf type degree Ia, IIa \& Ib in the right hip and in Graf type degree Ia, IIa \& IIb in the left hip. Clinical suspicion was more frequent in Graf type degree IIc. Positive family history \& oligohydramnios were the most frequent risk factors in Graf type IIb in the right hip. Graf type Ia was the most frequent degree among both males \& females in the studied group in the right and left hips. Among risk factors breech presentation and oligohydramnios were significantly higher in female infants than male infants while caesarean section was significantly higher in male infants than female infants. Normal degree of hip stability is the most frequent degree in the right and left hips by Harche dynamic technique.
\end{abstract}

Keywords: Musculoskeletal, Ultrasonography, Hip Dislocation

\section{Introduction}

Developmental dysplasia of the hip (DDH) is the most common congenital musculoskeletal disorder in infants and is characterized by varying displacement of the proximal femoral head from the acetabulum, with poor development of the acetabulum (1).

Developmental dysplasia of the hip is a term used to describe an abnormal relationship between the femoral head and the acetabulum (2).

Developmental dysplasia of the hip (DDH) varies in severity, ranging from neonatal hip instability with or without associated acetabular dysplasia to irreducible dislocation. In its severe form and even with surgical treatment, DDH results in shortening of the affected leg and early osteoarthritis (3).

In most cases there is only mild looseness of the hip joint. However some infants may be severely affected, having total dislocation of one or both of these joints. Hip dysplasia occurs in approximately 5 to 20 out of 1000 infants at birth. All infants should be checked shortly after birth for instability of the hip joint. If the diagnosis is missed shortly after birth, it may be more difficult to treat the disorder later. Hip dysplasia can be discovered by physical examination (4).

Clinical instability of the hip is the traditional hallmark of the disorder. In an unstable hip, the femoral head and acetabulum may not have a normal growth and may result 
in permanent disability. DDH can lead to premature degenerative joint disease, impaired walking, and chronic pain (5).

Ultrasonography (US) is considered the imaging technique of choice for the diagnosis of DDH in the neonate and infant (1).

Ultrasound is safe and noninvasive method of imaging of the hip. it can be used both for diagnosis and to monitor treatment. It provides advantages when combined with clinical examination. It can provide information on hip position, stability, and morphology and is more sensitive than clinical examination and therefore can be used to resolve the dilemma of whether to splint an unstable hip immediately or to delay treatment with the hope that transient instability will resolve spontaneously (2).

There is now ample evidence that ultrasonography can play a major role in the detection and management of developmental dysplasia of the hip in children younger than one year of age (2).

Hip ultrasonography has the ability not only to visualise the unossified parts of the newborn hip but also to show movement of the femoral head within the socket has brought about an enormous improvement in infant hip diagnosis. Hip ultrasound is practicable, reproducible, and able to be taught and learnt (5).

\section{Aim of the Work}

To assess the role of high resolution ultrasound in screening of the developmental dysplasia of the hip in the high risk infants group.

\section{Patients and Methods}

The current study included 270 cases of high risk infants for developmental dysplasia of the hip referred from the pediatric department \& pediatric out patient clinic to the Radiodiagnosis Department for ultrasonographic examination of their hip joints. They were (124) males and (146) females. Their ages ranged from 4 weeks to 6 months

Risk factors of developmental dysplasia of the hip include: Infants with a family history of hip dysplasia, breech presentation, oligohydramnios bicornuate uterus, large baby, maternal primiparity, caesarean section, excessive fetal moulding, clinical suspicion (neonatal hip instability /limb shortening/limitation of hip movement), female firstborn, other congenital anomalies as (foot deformity/ clubfoot/neonatal torticollis), twins

All infants were subjected to the following: history taking, clinical examination by pediatrician or pediatric orthopedic surgeon, ultrasonographic examination of both hips using high frequency linear transducer. Past history including previous limited hip joint movement or operations, perinatal history including prenatal history (maternal disease, oligohydramnios), natal history (time, type, site of delivery \& if complicated or not), postnatal (crying, cyanosis, jaundice, resuscitative measures) and family history of hip developmental dysplasia are also recorded .

Clinical examination: Local examination of the hip joint was performed to detect signs of developmental dysplasia of the hip as (limited abduction $<70$ degrees -asymmetric skin folds - shortened thigh on the side of the dislocated hip - loss of normal mild hip/knee flextion) and external signs like swelling or deformity, detection of areas of tenderness. N.B. Technical tips : Feed baby 3 hours before ultrasound examination, feed during examination, beware of getting wet, foot pedal capability.

Ultrasound examination: Ultrasound examination was performed with 5 or $7.5 \mathrm{MHz}$ linear probe of HITACHI (EU-7000) apparatus - FUKUDA DENSHI (UF-850XTD) apparatus using: the Graf static method: The infant was examined in the decubitus position, hips and knees slightly flexed. In coronal view the standard plane is best defined when: a straight iliac bony interface, parallel to the transducer, a bony acetabular promontory, with bright echoes at the lower end, the cartilaginous acetabular roof with an echogenic tip (fibrocartilaginous tip) at the point of the labrum. The angle subtended by reference lines through the iliac bone and tangential to the osseous roof of the acetabulum, referred to as the alpha $(\alpha)$ angle, represents the hard bony roof and reflects the depth of the acetabulum and values of the angle were recorded. A second angle, the beta $(\beta)$ angle, subtended by a line drawn through the labrum and intersecting the iliac reference line, represents the cartilaginous roof of the acetabulum and indirectly reflects the position of the femoral head and values of the angle were recorded. Fig 1

The Harcke dynamic technique: has four steps: Coronal neutral: The infant was examined in the decubitus position, hips and knees slightly flexed. Probe placed in coronal view and the image obtained. Coronal flexion: the hip in $90^{\circ}$ flexion and Probe placed in coronal view. The triradiate cartilage is identified and then the transducer is moved posteriorly and anteriorly. When the transducer is posterior to the triradiate cartilage the femoral head should not normally be visible, and if seen it indicates that the head is abnormally sited .Then by gently pushing and pulling the knee as in the Barlow maneuver (downward force while the hip is adducted) and comment on state of hip joint (normal - subluxable -subluxated - dislocatable - dislocated). Transverse neutral: The infant was examined in the decubitus position with slightly flexed hip (neutral position) and the hip scanned transversely. The image obtained is the acetabulum centered at the level of the triradiate cartilage. The normal femoral head is located within the acetabulum, with the center of the head at this level as Lollipop on a stick or Golf ball on T. When dislocated the triradiate cartilage cannot be seen in this plane. Transverse flexion: The infant was placed in an oblique position with the hip in $90^{\circ}$ flexion. The hip is scanned transversely. This produces a ' $U$ ' configuration with the femoral capital epiphysis central to the femoral metaphysis and the ischium. When displaced the head cannot be seen within the ' $U$ '. On 
dynamic scanning with gentle posterior pressure on the knee, if the hip is unstable then the gap between the head and the acetabulum increases. Conversely with the hip in abduction and gently pulling on the knee, the dislocated head can be relocated within the acetabulum.

Statistical analysis: The data collected were tabulated and

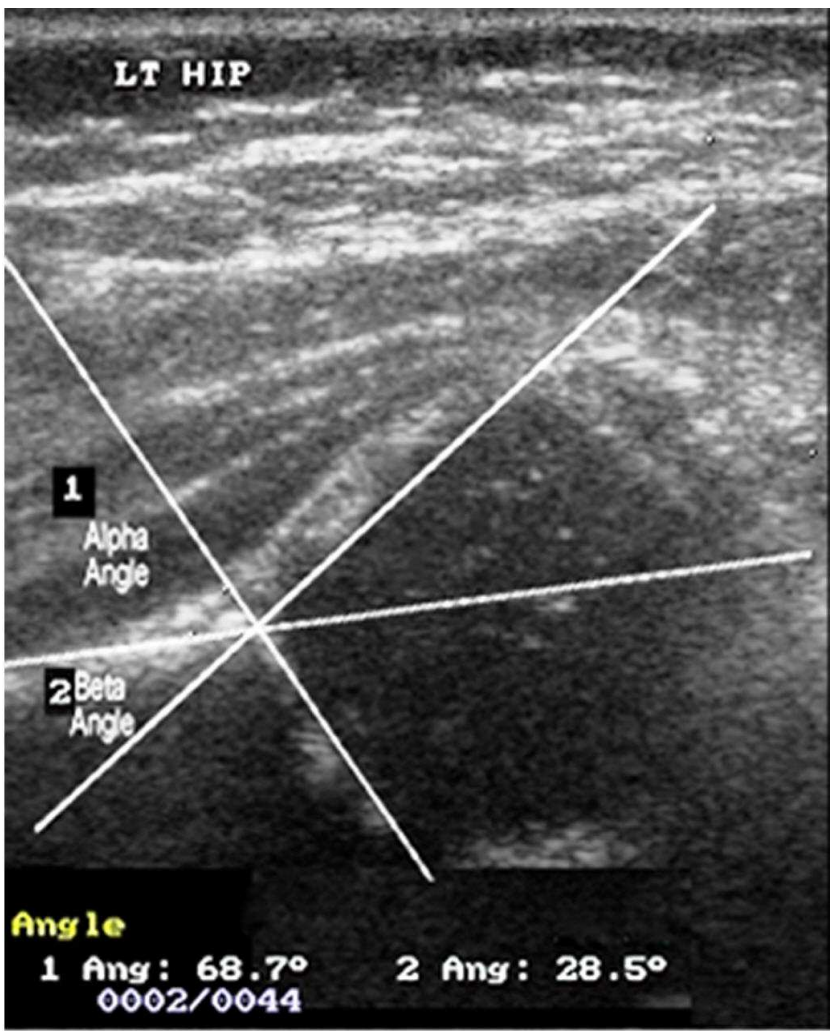

(A) analyzed by SPSS (statistical package for social science) version 17.0. Two types of statistics were done: Descriptive statistics: percentage (\%), mean (x) and standard deviation(SD). Analytic statistics: Chi-squre test $\left(\mathrm{x}^{2}\right)$ : was used to study association between two qualitative variables. Fig 2.

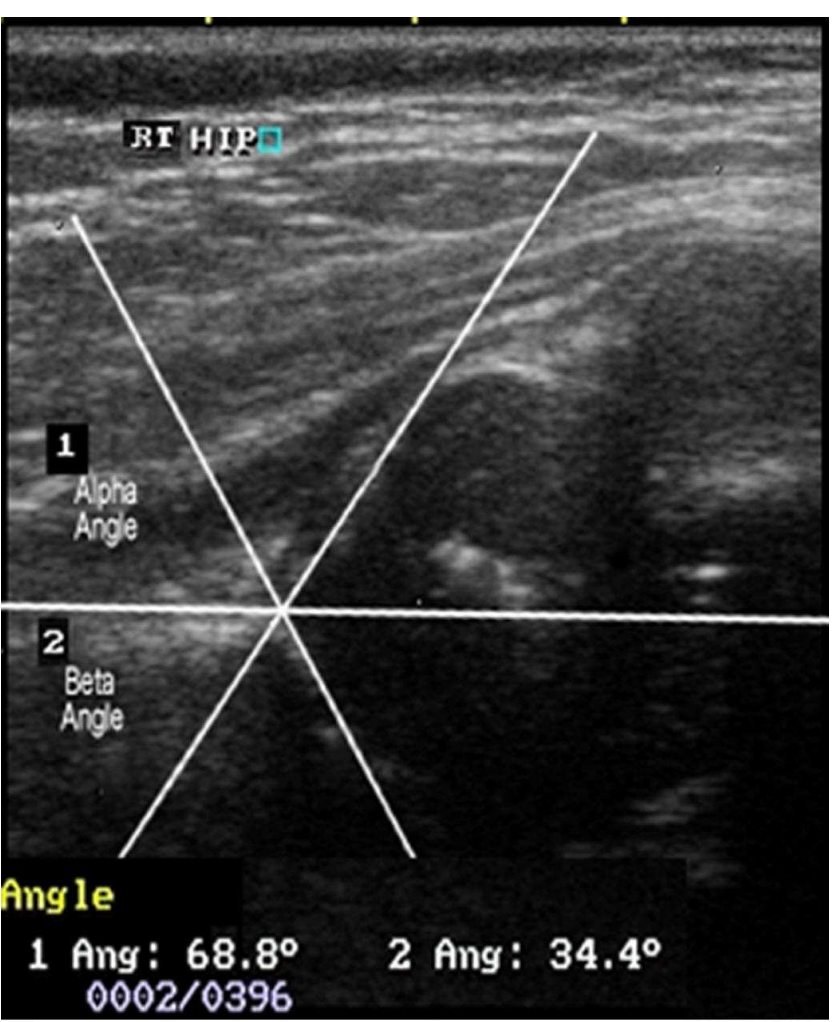

(B)

Fig. (1). Male infant aged 3.5 monthes.

Risk factor: breech presentation

Ultrasonographic findings:

Lt hip:

Alpha angle $=68.7^{\circ}$

Beta angle $=28.5^{\circ}$

Rt hip:

Alpha angle $=68.8^{\circ}$

Beta angle $=34.4^{\circ}$

Ultrasonographic type according to Graf: Rt hip : type Ia - Lt hip : type Ia After dynamic technique : Rt hip: normal - Lt hip:normal 


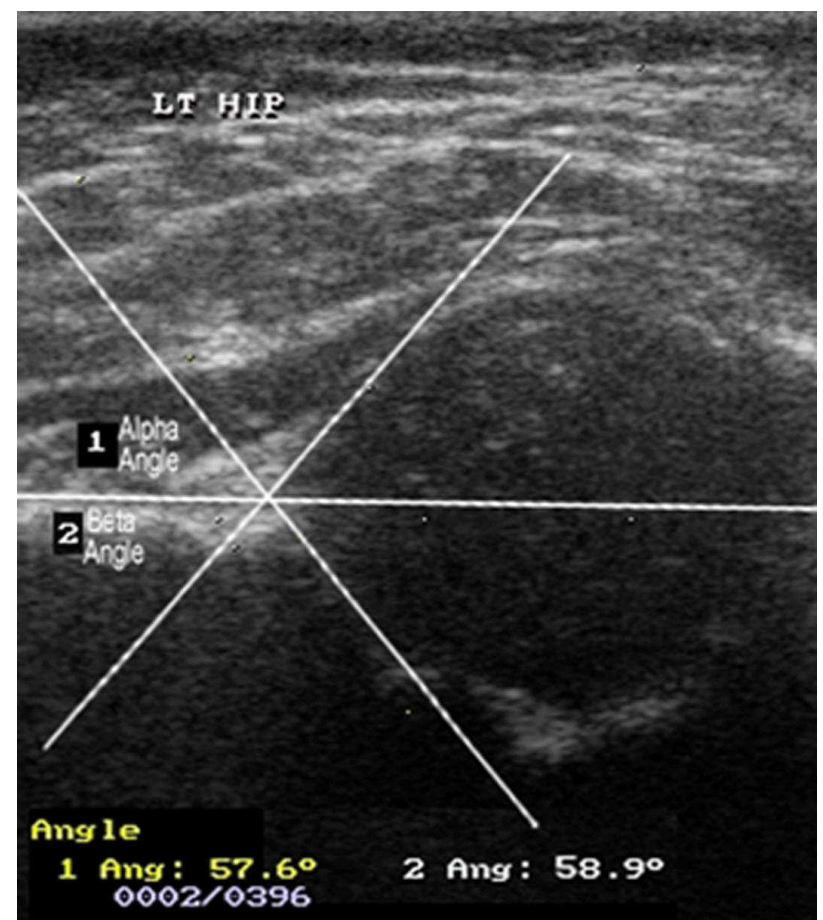

(A)

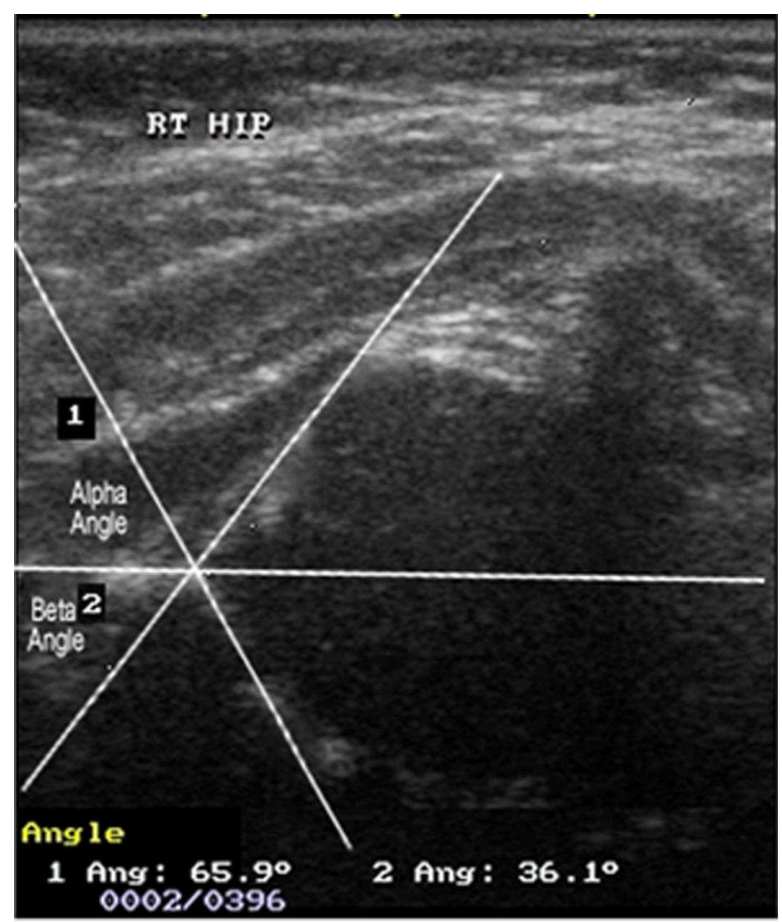

(B)

Fig. (2). Male infant aged 4 months.

Risk factor: caesarean section delivery

Ultrasonographic findings :

Lt hip:

Alpha angle $=57.6^{\circ}$

Beta angle $=58.9^{\circ}$

Rt hip:

Alpha angle $=65.9^{\circ}$

Beta angle $=36.1^{\circ}$

Ultrasonographic type according to Graf: Rt hip : type Ia - Lt hip : type IIb

after dynamic technique : Rt hip: normal - Lt hip: subluxable

\section{Results}

Out of the studied 270 cases of high risk infants for DDH, the results by ultrasound showed that $46 \%$ of studied group were males and $54 \%$ were females, mean age is $4.01+1.55$ mo. The most prevalent risk factor in our group was ceaserean section delivery (52\%) Followed by breech presentation and oligohydramnios (14\% for each).

This illustrated in the following table and chart. Table (1), Fig (3)

Table (1). Prevalence of different risk factors among studied group.

\begin{tabular}{lll}
\hline Risk factor & No & \% \\
\hline Caesarean section delivery & 140 & 52 \\
Breech presentation & 38 & 14 \\
Oligohydramnios & 38 & 14 \\
Clinical suspicion & 16 & 6 \\
Twins & 16 & 6 \\
Positive family history & 16 & 6 \\
$1^{\text {st }}$ born/CS (combined risk factors) & 6 & 2 \\
total & 270 & 100 \\
\hline
\end{tabular}

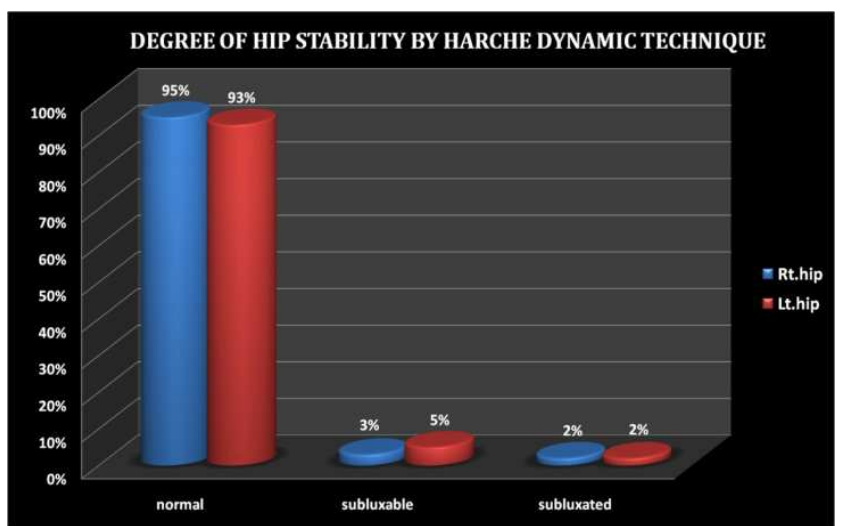

Fig. (3). Degree of Hip Stability by Harche Dynamic Technique

The mean value of Alpha angle of the right hip was high than that of the left hip, where mean values of Beta angle of the left hip was high than that of the right hip. These findings are illustrated in the following table. Table (2) 
Table (2). Mean value of ultrasound Alpha \& Beta angles among the studied roup.

\begin{tabular}{llll}
\hline U/S scan & & Mean + SD & Range \\
\hline \multirow{2}{*}{ Alpha angle } & RT hip & $64.9+8.19$ & $42^{\circ}-83^{\circ}$ \\
& LT hip & $62.41+6.63$ & $47^{\circ}-80^{\circ}$ \\
\multirow{2}{*}{ Beta angle } & RT hip & $36.34+13.14$ & $20^{\circ}-71^{\circ}$ \\
& Lt hip & $41.45+15.78$ & $18^{\circ}-96^{\circ}$ \\
\hline
\end{tabular}

Graf type degree Ia was the most frequent degree in the right \& left hips followed by IIa in right hip and IIb in left hip. These findings are illustrated in the following table and chart. Table (3), Fig (4)

Table (3). Number \& percentage distribution of Graf type degree among the studied group.

\begin{tabular}{|c|c|c|c|c|c|c|c|c|c|c|}
\hline \multirow{2}{*}{$\begin{array}{l}\text { Graf } \\
\text { type } \\
\text { degree }\end{array}$} & \multicolumn{2}{|l|}{ Ia } & \multicolumn{2}{|l|}{ Ib } & \multicolumn{2}{|l|}{ IIa } & \multicolumn{2}{|l|}{ IIb } & \multicolumn{2}{|l|}{ IIc } \\
\hline & No & $\%$ & No & $\%$ & No & $\%$ & No & $\%$ & No & $\%$ \\
\hline Rt.hip & 222 & 82.1 & 5 & 1.9 & 27 & 10 & 11 & 4.1 & 5 & 1.9 \\
\hline Lt.hip & 205 & 76 & 0 & 0 & 22 & 8.1 & 43 & 15.9 & 0 & 0 \\
\hline
\end{tabular}

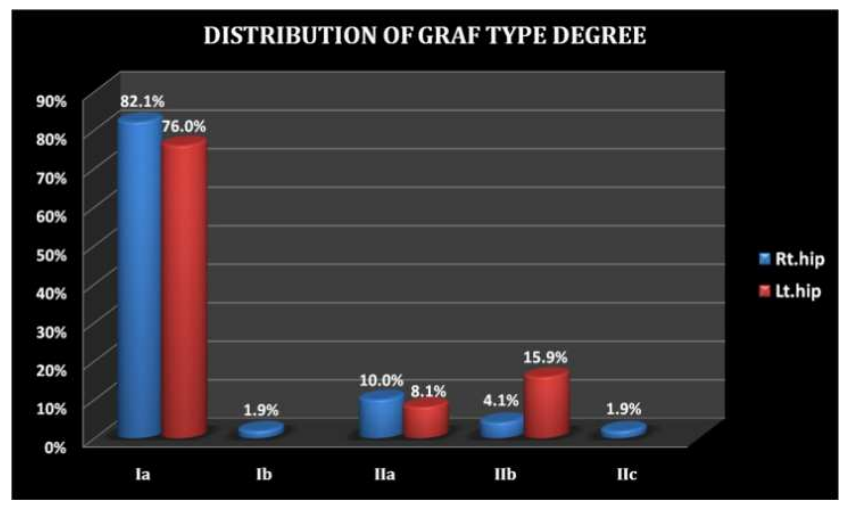

Fig. (4). Distribution of Graf Type Degree

For the right hip caesarean section delivery was the most prevalent risk factor in Graf type degree Ia, Ib \& IIa (53.7\%, $100 \%$ \& $60 \%$ respectively). While clinical suspicion was more frequent in Graf type degree IIc (100\%), where positive family history \& oligohydramnios were the most frequent risk factor in Graf type IIb (50\% for each). These findings are illustrated in the following table.Table (4), Fig (5)

Table (4). Relationship between risk factors \& Graf type degree in right hip.

\begin{tabular}{|c|c|c|c|c|c|c|c|c|c|c|}
\hline Risk factor & Ia & & Ib & & IIa & & IIb & & IIc & \\
\hline & No & $\%$ & No & $\%$ & No & $\%$ & No & $\%$ & No & $\%$ \\
\hline Caesarean section delivery & 120 & 53.7 & 5 & 100 & 18 & 60 & 0 & 0 & 0 & 0 \\
\hline Breech presentation & 32 & 14.6 & 0 & 0 & 5 & 20 & 0 & 0 & 0 & 0 \\
\hline Oligohydramnios & 27 & 12.2 & 0 & 0 & 5 & 20 & 5 & 50 & 0 & 0 \\
\hline Twins & 16 & 7.3 & 0 & 0 & 0 & 0 & 0 & 0 & 0 & 0 \\
\hline Clinical suspicion & 11 & 4.9 & 0 & 0 & 0 & 0 & 0 & 0 & 5 & 100 \\
\hline Positive family history & 11 & 4.9 & 0 & 0 & 0 & 0 & 5 & 50 & 0 & 0 \\
\hline $\begin{array}{l}1^{\text {st }} \text { born/ Caesarean section delivery } \\
\text { (combined risk factors) }\end{array}$ & 5 & 2.4 & 0 & 0 & 0 & 0 & 0 & 0 & 0 & 0 \\
\hline total & 222 & 100 & 5 & 100 & 28 & 100 & 10 & 100 & 5 & 100 \\
\hline
\end{tabular}

For the left hip caesarean section delivery was the most prevalent risk factor in Graf type degree Ia, IIa \& IIb (55.3\%,

$50 \% \& 37.2 \%$, respectively).These findings are illustrated in the following table. Table (5)

Table (5). Relationship between risk factors \& Graf type degree in left hip in the studied group.

\begin{tabular}{|c|c|c|c|c|c|c|}
\hline \multirow{2}{*}{ Risk factor } & \multicolumn{2}{|l|}{ Ia } & \multicolumn{2}{|l|}{ IIa } & \multicolumn{2}{|l|}{ IIb } \\
\hline & No & $\%$ & No & $\%$ & No & $\%$ \\
\hline Caesarean section delivery & 114 & 55.3 & 11 & 50 & 16 & 37.2 \\
\hline Breech presentation & 27 & 13.2 & 0 & 0 & 11 & 25.6 \\
\hline Oligohydramnios & 16 & 7.9 & 11 & 50 & 11 & 25.6 \\
\hline Twins & 16 & 7.9 & 0 & 0 & 0 & 0 \\
\hline Positive family history & 16 & 7.9 & 0 & 0 & 0 & 0 \\
\hline Clinical suspicion & 11 & 5.3 & 0 & 0 & 5 & 11.6 \\
\hline $1^{\text {st }}$ born/ Caesarean section (combined risk factors) & 5 & 2.3 & 0 & 0 & 0 & 0 \\
\hline Total & 205 & 100 & 22 & 100 & 43 & 100 \\
\hline
\end{tabular}

For the right hip the Graf type Ia was the most frequent 
(91.3\% \& 74.7\%, respectively). These findings are illustrated

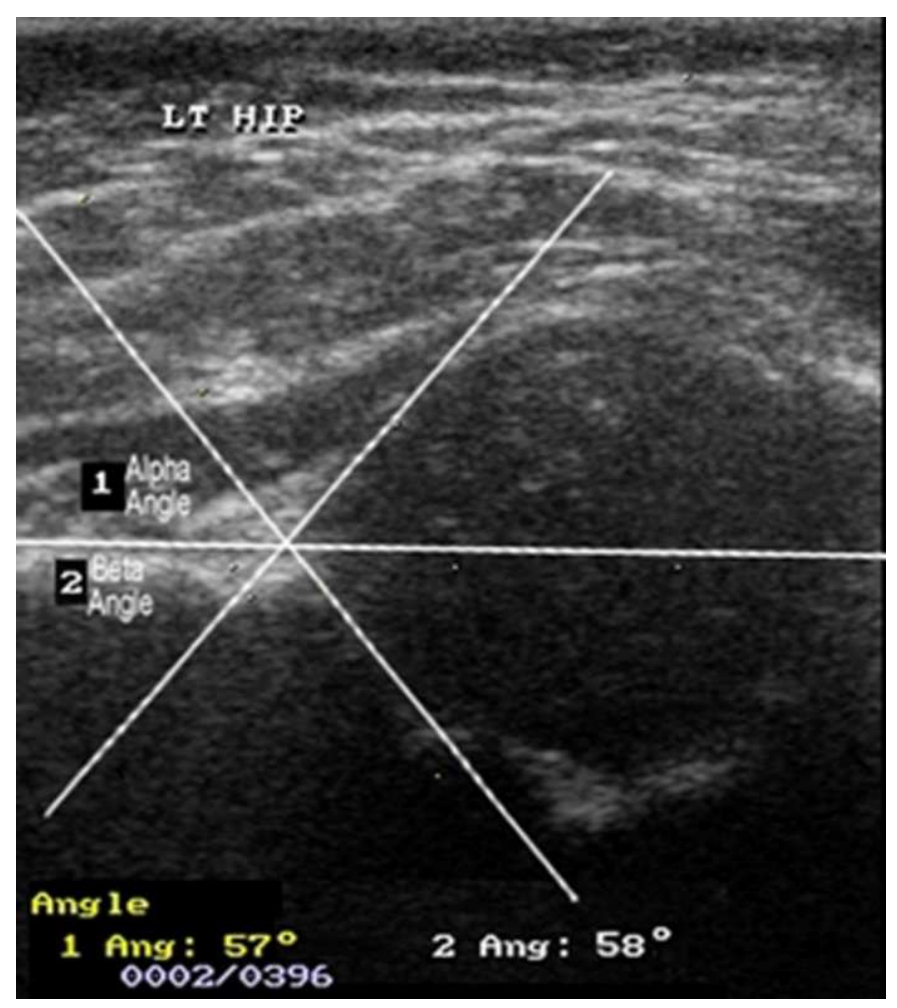

(A) in the following table. Table (6)

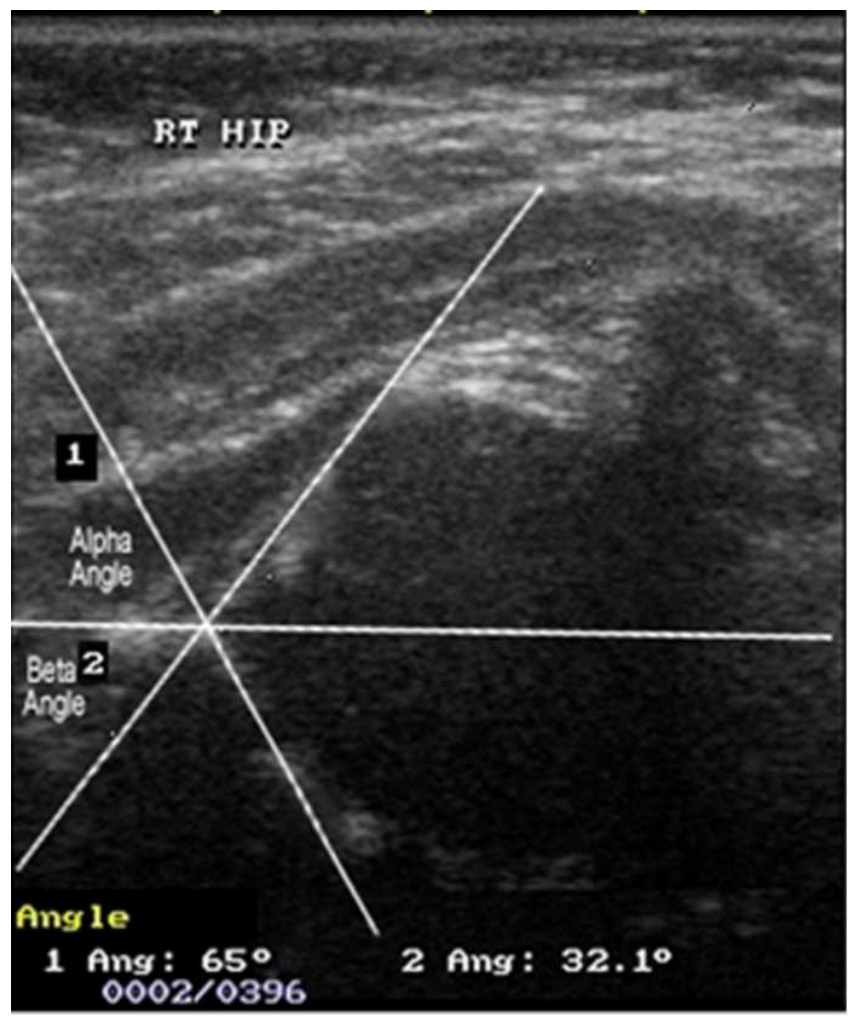

(B)

Fig. (5). Male infant aged 5 months.

Risk factor: twins

Ultrasonographic findings :

Lt hip:

Alpha angle $=57^{\circ}$

Beta angle $=58^{\circ}$

Rt hip:

Alpha angle $=65^{\circ}$

Beta angle $=32.1^{\circ}$

Ultrasonographic type according to Graf: Rt hip : type Ia - Lt hip : type IIb

After dynamic technique : Rt hip: normal - Lt hip: subluxable

Table (6). Relationship between sex \& Graf degree of right hip in the studied group.

\begin{tabular}{lllllll}
\hline \multirow{2}{*}{ Graf type degree } & Male & \multicolumn{3}{c}{ Female } & \multicolumn{3}{c}{ Total } \\
\cline { 2 - 7 } & No & $\mathbf{\%}$ & No & \% & No & \% \\
\hline Ia & 113 & 91.3 & 109 & 74.7 & 222 & 82.2 \\
Ib & 0 & 0 & 5 & 3.4 & 5 & 1.9 \\
IIa & 11 & 8.7 & 16 & 11.0 & 27 & 10 \\
IIb & 0 & 0 & 11 & 7.5 & 11 & 4.0 \\
IIc & 0 & 0 & 5 & 3.4 & 5 & 1.9 \\
Total & 124 & 100 & 146 & 100 & 270 & 100 \\
\hline
\end{tabular}

For the left hip Graf type Ia was the most frequent degree among both females \& males $(83.1 \% \& 70.5 \%$ respectively) These findings are illustrated in the following table. Table (7), Fig 6
Table (7). relationship between sex \& Graf degree of left hip in the studied group.

\begin{tabular}{lllllll}
\hline \multirow{2}{*}{ Graf type degree } & \multicolumn{2}{l}{ Male } & \multicolumn{3}{c}{ Female } & \multicolumn{3}{c}{ Total } \\
\cline { 2 - 7 } & No & $\%$ & No & $\%$ & No & $\%$ \\
\hline Ia & 103 & 83.1 & 103 & 70.5 & 206 & 76.3 \\
IIa & 5 & 4.0 & 16 & 11.0 & 21 & 7.8 \\
IIb & 16 & 12.9 & 27 & 18.5 & 43 & 15.9 \\
Total & 124 & 100 & 146 & 100 & 270 & 100 \\
\hline
\end{tabular}

Among risk factors breech presentation and oligohydramnios were significantly higher $(\mathrm{P}<0.001)$ in female infants than male infants while caesarean section was significantly higher $(\mathrm{P}<0.001)$ in male infants than female infants. This is illustrated in the following table. Table (8) 
Table (8). Comparison between male \& female infants regarding risk factors.

\begin{tabular}{|c|c|c|c|c|c|c|}
\hline \multirow{2}{*}{ Risk factor } & \multicolumn{2}{|c|}{ Male } & \multicolumn{2}{|c|}{ Female } & \multirow{2}{*}{$\mathbf{X}^{2}$} & \multirow{2}{*}{$\begin{array}{l}\text { P- } \\
\text { Value }\end{array}$} \\
\hline & No & $\%$ & No & $\%$ & & \\
\hline Oligohydramnios & 5 & 4.3 & 32 & 22.0 & 13.18 & $<0.001$ \\
\hline $\begin{array}{l}\text { Caesarean section } \\
\text { delivery }\end{array}$ & 82 & 66.3 & 59 & 40.4 & 13.9 & $<0.001$ \\
\hline Clinical suspicion & 11 & 8.7 & 5 & 3.4 & 2.19 & 0.13 \\
\hline Breech presentation & 4 & 3.3 & 35 & 24 & 16.3 & $<0.001$ \\
\hline Twins & 11 & 8.7 & 5 & 3.4 & 2.19 & 0.13 \\
\hline $\begin{array}{l}1^{\text {st }} \text { born/caesarean } \\
\text { section (combined } \\
\text { risk factors) }\end{array}$ & 0 & 0 & 5 & 3.4 & 3.4 & 0.06 \\
\hline $\begin{array}{l}\text { Positive family } \\
\text { history }\end{array}$ & 11 & 8.7 & 5 & 3.4 & 2.19 & 0.13 \\
\hline Total & 124 & 100 & 146 & 100 & & \\
\hline
\end{tabular}
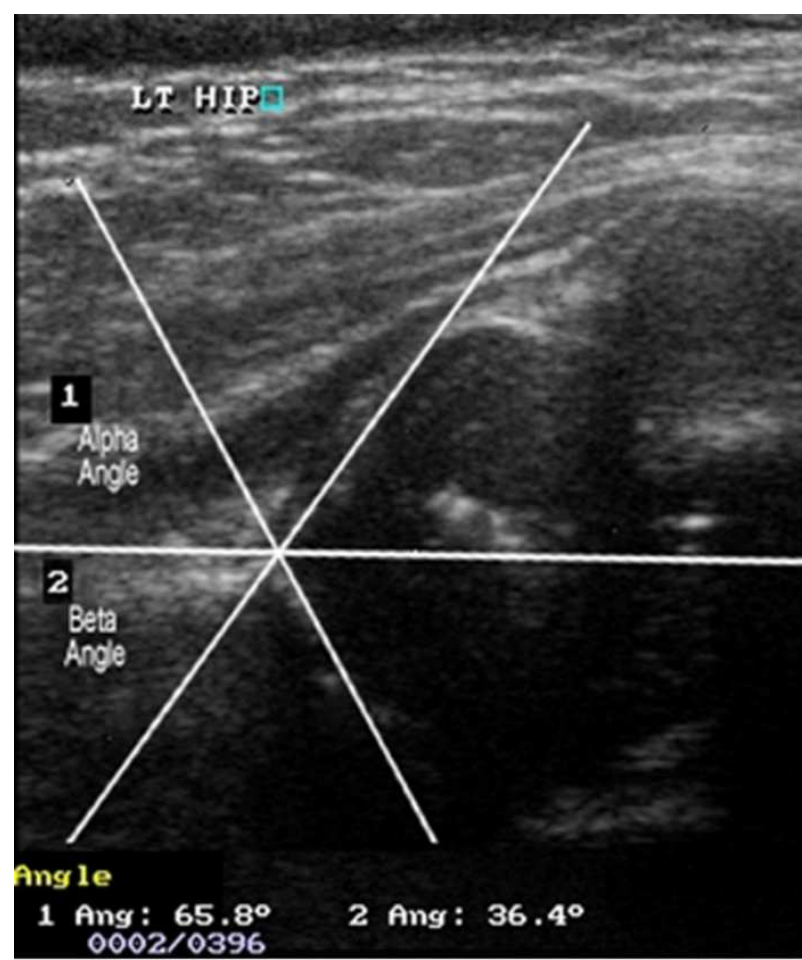

(A)
By Harche dynamic technique the normal degree of the hip stability was the most frequent degree in the right \& left hips. This is illustrated in the following table and chart. Table (9), Fig 7

Table (9). Number \& percentage distribution of degree of hip stability by Harche dynamic technique among the studied group.

\begin{tabular}{lllllll}
\hline \multirow{2}{*}{ Hip stability degree } & normal & \multicolumn{3}{c}{ subluxable } & \multicolumn{2}{c}{ subluxated } \\
\cline { 2 - 7 } & No & \% & No & \% & No & \% \\
\hline Rt.hip & 257 & 95 & 8 & 3 & 5 & 2 \\
Lt.hip & 251 & 93 & 14 & 5 & 5 & 2 \\
\hline
\end{tabular}$$
\text { (1) }
$$

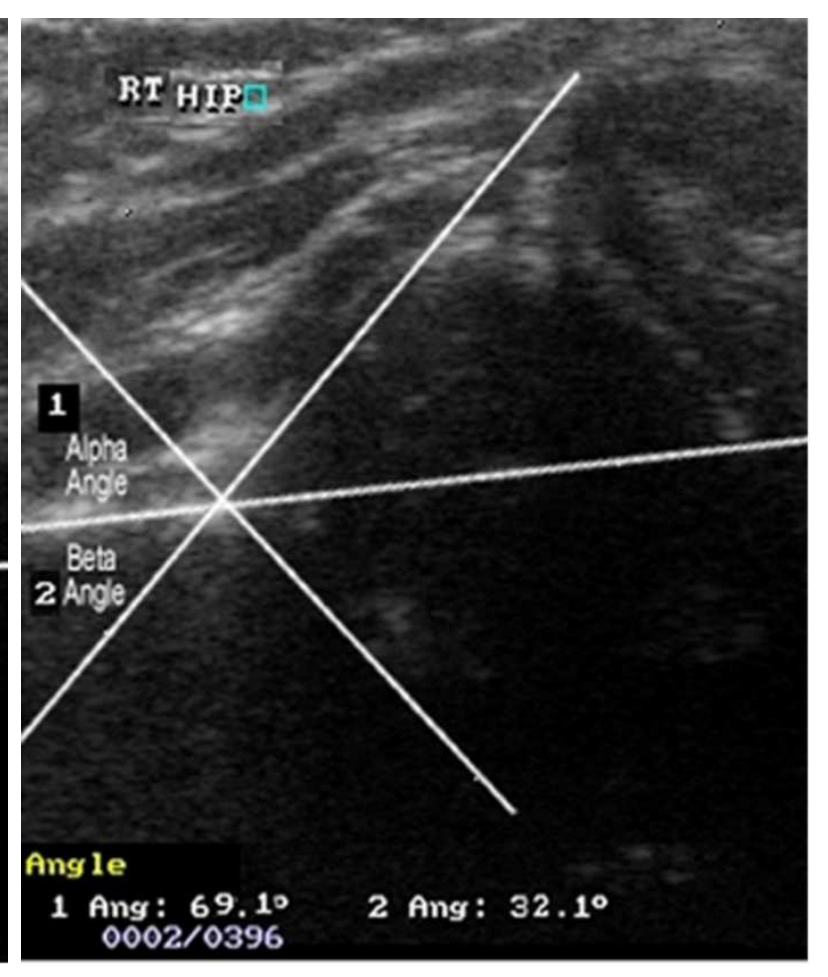

(B)

Fig. (6). Female infant aged 5 months.

Risk factor: caesarean section delivery

Ultrasonographic findings :

Lt hip:

Alpha angle $=65.8^{\circ}$

Beta angle $=36.5^{\circ}$

Rt hip:

Alpha angle $=69.1^{\circ}$

Beta angle $=32.1^{\circ}$

Ultrasonographic type according to Graf: Rt hip: type Ia - Lt hip: type Ia

After dynamic technique : Rt hip: normal - Lt hip: normal 


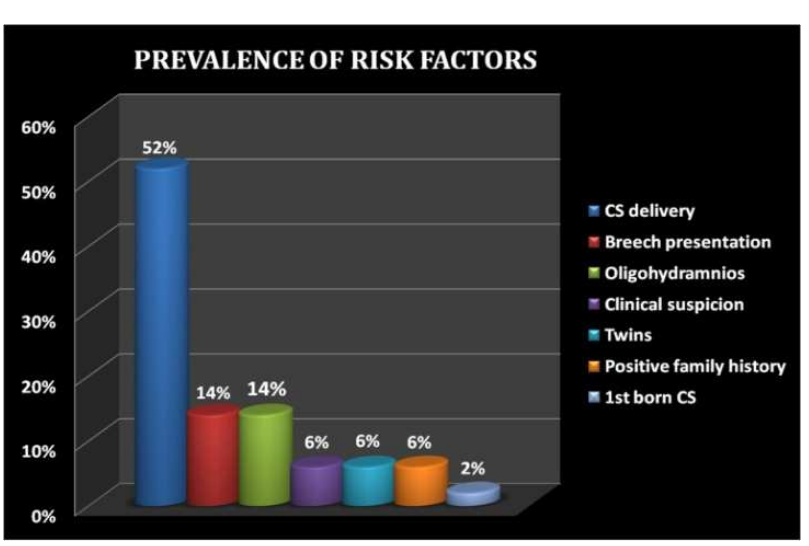

Fig. (7). Prevalence of Risk Factors

\section{Discussion}

Developmental dysplasia of the hip (DDH) is the most common congenital musculoskeletal disorder in infants and is characterized by varying displacement of the proximal femoral head from the acetabulum, with poor development of the acetabulum (1).

Developmental dysplasia of the hip (DDH) varies in severity, ranging from neonatal hip instability with or without associated acetabular dysplasia to irreducible dislocation. In its severe form and even with surgical treatment, DDH results in shortening of the affected leg and early osteoarthritis (3).

Ultrasonography (US) is considered the imaging technique of choice for the diagnosis of DDH in the neonate and infant (1)

Sonographic screening of all newborns identify cases at an earlier age and lead to earlier treatment.(6).

We demonstrated in the present study that $(21.9 \%)$ of studied group with Graf type-II of right hip and (29.5\%) with Graf type-II of left hip were females and $(8.7 \%)$ of studied group with Graf type-II of right hip and (16.9\%) with Graf type-II of left hip were males (females more affected than males).

These results were in agreement with Paton, 2004 (7) who found that incidence of DDH in females seven times to males Weinstein, 2001 (8) who reported that $80 \%$ of infants with DDH are females. This is postulated to be related to the effects of additional estrogen produced by the female fetus, which increases ligamentous laxity. as well as Jones and Powell, 1990 (9) who found that females are more affected than males.

In the present study we found that: For the right hip: (91.3\%) male infants were with Graf type-I and (8.7\%) were with Graf type-II, while (78.1\%) female infants were with Graf type-I and $24(21.9 \%)$ were with Graf type-II. For the left hip: (83.1\%) male infants were with Graf type-I and $(16.9 \%)$ were with Graf type-II, while $(70.5 \%)$ female infants were with Graf type-I and (29.5\%) were with Graf type II. This means that Graf type- I is the most frequent degree among both males and females.

This was in agreement with Dante et al., 1997 (10) who demonstrated that the frequency of a normal finding (type I) varied from 37.3 to $72.1 \%$; that of type IIa, from 23.6 to $57.6 \%$; that of type IIc-IId, from 0.8 to $7.0 \%$; and that of type III-IV, from 0.0 to $1.1 \%$, Roovers, 2005 (11) who demonstrated that the frequency of (type I) $62.4 \%$, (type IIa) $32 \%$, (type IIb) $1.9 \%$, (type IIc) 0.7 , (type III) $0.7 \%$, (type IV) $0.02 \%$ as well as Jones and Powell, 1990 (9) who reported that the frequency of (type I) $75 \%$, (type II) $14 \%$, (type III) $8 \%$, (type IV) $1.5 \%$.

Our study demonstrated that prevalence of the different risk factors among studied group was (13.7\%) for Oligohydramnios, (52.2\%) for Caesarean section delivery, (6\%) for Clinical suspicion, (14.4\%) for breech presentation, (6\%) for Twins, (2\%) for $1 \mathrm{st}$ born/CS (combined risk factors) and $(6 \%)$ for Positive family history. This means that the most prevalent risk factor in our group was Caesarean section delivery (52.2\%). Fig 8

This was in agreement with Sutton, 2003 (12) who found that children born by caesarean section are more likely to have associated instability and dislocations and in disagreement with Dante et al., 1997 (10) who reported that the most frequent risk factor was family history followed by breech presentation and oligohydramnios

In the present study, we found that among the risk factors breech presentation, and oligohydramnios were significantly higher in female infants than male infants while caesarean section was significantly higher in male infants than female infants.

This was in agreement with Paton et al., 1999 (13) who demonstrated that $60 \%$ of dislocations were associated with at-risk factors, including breech delivery, caesarean section, oligohydramnios.

In our study, we found that values of alpha angle of right hip is high than that of left hip, while values of beta angle of left hip is high than that of right hip (left hip more affected than right hip).

In our study, we found that $(16 \%)$ of right hips of studied group were with Graf type- II and (23.7\%) of left hips of studied group were with Graf type- II (left hip more affected than right hip).

This was in agreement with Storer and Skaggs, 2006 (14) who said that left hip is affected in $60 \%$ of infants, the right hip in $20 \%$, and both hips in $20 \%$ as in the most common intrauterine position left hip is adducted against the mother's lumbosacral spine and also in agreement with Jones and Powell (9) who reported that left side is more affected than right side.

In the present study, we found that caesarean section delivery is the most prevalent risk factor in Graf type degree Ia, IIa \& Ib $(53.7 \%, 60 \%$ \& 100\%, respectively) in Rt hip and in Graf type degree Ia, IIa \& IIb $(55.3 \%, 50 \%$ \& $37.2 \%$, respectively) in left hip. While clinical suspicion is more frequent in Graf type degree IIc (100\%), where positive family history \& oligohydraminos are the most frequent risk factor in Graf type IIb (50\% for each) in right hip.

This was in disagreement with Walter et al., 1992 (15) who found that there is an association between type III or IV hip 
and female gender (relative risk $=2.8$ ), family history of DDH (relative risk $=3.4$ ), and breech presentation((relative risk $=2.8$ ). and A weaker association with type IIc and IId hip was also detected. It may be due to low number of sample in our study.

In our study, we demonstrated that the normal degree of the hip stability is the most frequent degree in the right \& left hips by Harche dynamic technique.

This was in agreement with Marks et al, 1994 (16) who

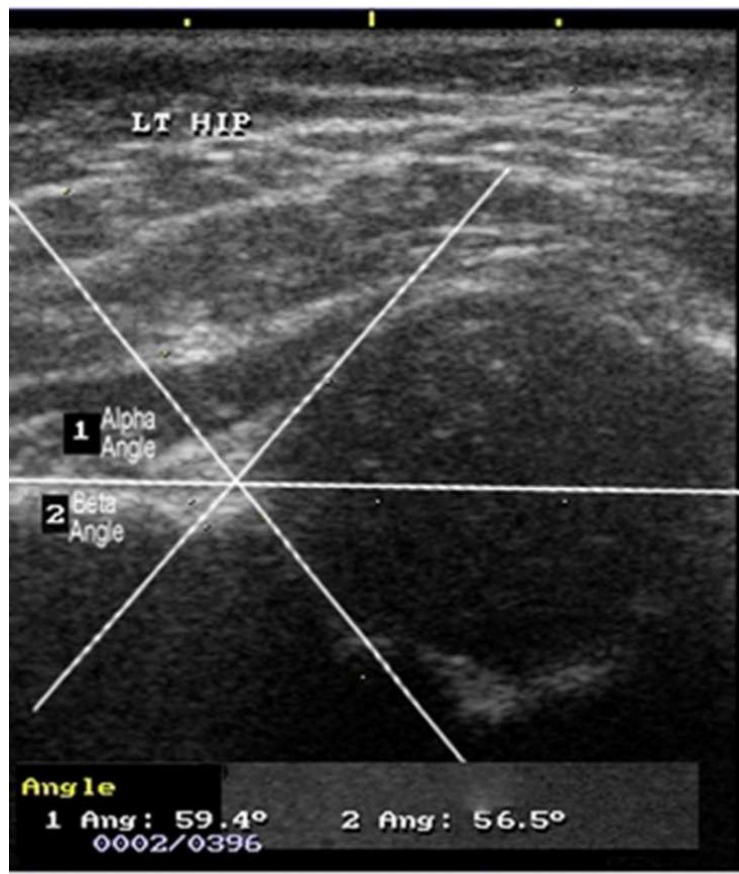

(A) reported that 10 percent of (847) infants had abnormal scans, but 90 percent had normal scans by the time that they were nine weeks of age.

We recommend ultrasound screening of DDH should be done for all high risk infants, It is better to do examination after 4-6 weeks as before that will often reveal minor degrees of dysplasia that resolve spontaneously and do not need treatment, It is recommended to do both Graf static method and Harche dynamic technique.

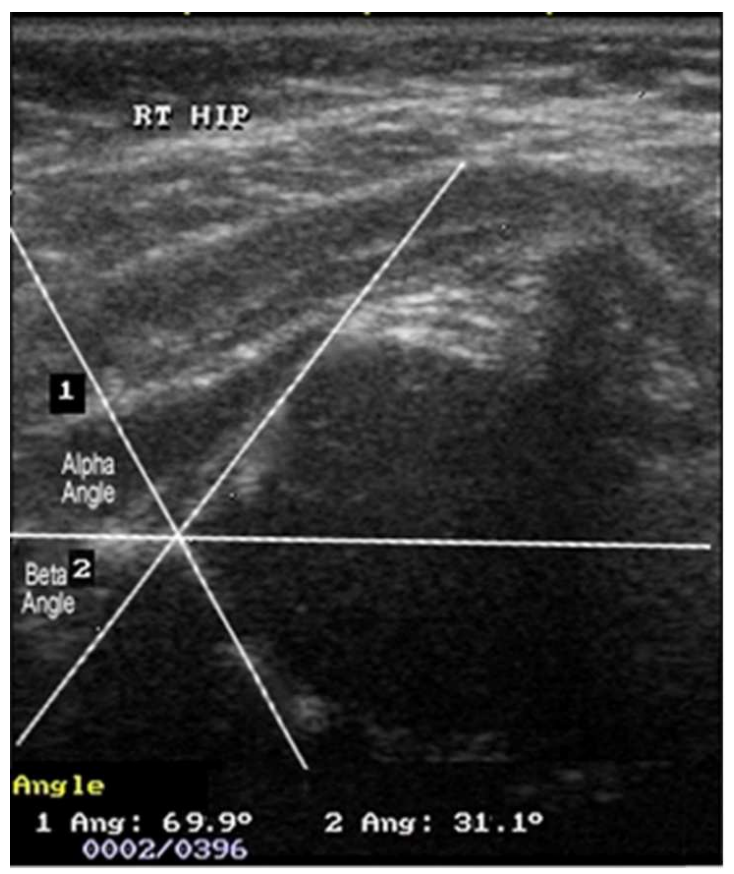

(B)

Fig. (8). Male infant aged 6 months.

Risk factor: caesarean section delivery

Ultrasonographic findings:

Lt hip:

Alpha angle $=59.4^{\circ}$

Beta angle $=56.5^{\circ}$

Rt hip:

Alpha angle $=69.9^{\circ}$

Beta angle $=31.1^{\circ}$

Ultrasonographic type according to Graf: Rt hip : type Ib - Lt hip : type IIa

After dynamic technique : Rt hip: normal - Lt hip: normal

\section{References}

[1] Herring JA. Developmental dysplasia of the hip. In Tachdjian's pediatric orthopedics. Edited by Herring JA, W.B. Saunders co. Philadelphia 2002; 513-646.

[2] Wientroub S and Grill F. Ultrasonography in Developmental Dysplasia of the Hip, J. Bone Joint Surg Am.2000; 82:1004.

[3] Karen R, Carol D, Kari R, et al. Immediate Treatment Versus Sonographic Surveillance for Mild Hip Dysplasia in Newborns, j. of Pediatrics. 2010; 125:e9-e16

[4] Storer D. How is hip dysplasia diagnosed? Am Fam Physician.2006; 74 (8):1310-6.

[5] Scott S, Lercher K, Benaroya A, et al. Diagnosis and management of infant hip dysplasia.In: hip ultrasonography edited by Graf R. Second edition, Library of Congress Control Number.2006 p. 6-8.

[6] Tonnis D, Storch K and Ulbrich H. Results of newborn screening of $\mathrm{CDH}$ with and without sonography and correlation of risk factors. J. Pediatr Orthop.1990; 10: 145-52.

[7] Paton RW, Hopgood PJ and Eccles K. Instability of the neonatal hip: the role of early or late splintage. International Orthopaedics. 2004; 28, 5: 270-73.

[8] Weinstein S. Developmental hip dysplasia and dislocation. In: pediatric orthopaedics ed. by Morrissy RT, Weinstein SL, Lovell and Winter's. Philadelphia: Lippincott Williams and Wilkins. 2001; chapter 2, p. 905-56. 
[9] Jones DA and Powell N. Ultrsound and neonatal hip screening British editional society of bone and joint surgery.1990; 72B:457-9

[10] Dante B, Giuseppe A, Francesco A, et al. Screening for developmental dysplasia of the hip. Pediatrics: the official journal of the American Academy. 1997; e5: 99

[11] Roovers EA, Boere-Boonekamp MM, Castelein RM, et al. Effectiveness of ultrasound screening for developmental dysplasia of the hip, Arch Dis Child Fetal Neonatal Ed.2005; 90:f25-f30

[12] Sutton D. Developmental dysplasia of the hip, British Journal of Radiology. 2003; 7:1856
[13] Paton RW, Srinivasan MS, Shah B and Hollis S. Ultrasound screening for hips at risk in developmental dysplasia: is it worth it? J. Bone Joint Surg [Br]. 1999; 81-B: 255-8.

[14] Storer S and Skaggs D. Developmental dysplasia of the hip. Annual Clinical Focus on caring for children and adolescents. ACF;Am Fam Physician. 2006; 74(8):1310-1316.

[15] Walter RS, Donaldson JS, Davis CL, et al. Ultrasound screening of high risk infants. A method to increase early detection of congenital dysplasia of the hip. Am J Dischild. 1992; 146:230-34

[16] Marks DS, Clegg J and Al-Chalabi AN. Routine ultrasound screening for neonatal hip instability. J. Bone and Joint Surg.1994; 76-B(4): 534-38 